\title{
SIW Cavity-backed Slot (Multi-)Antenna Systems for the Next Generation IoT Applications
}

\author{
S. Lemey *, O. Caytan *, D. Vande Ginste *, P. Demeester *, H. Rogier *, and M. Bozzi * \\ \# Dept. of Information Technology, Ghent University/iMinds, Ghent, Belgium \\ ${ }^{*}$ Dept. of Electrical, Computer and Biomedical Engineering, University of Pavia, Pavia, 27100, Italy
}

\begin{abstract}
Substrate integrated waveguide (SIW) cavitybacked slot antenna topologies are promising candidates to adress the specific design challenges posed by the Internet of Things (IoT). In this contribution, we demonstrate their potential by discussing two designs on two different, application-specific, innovative substrate materials. First, a compact, ultra-wideband three-element array with very low mutual coupling is presented for integration into furniture. In the second design, the half-mode SIW technique is applied to obtain a miniaturized ultra-wideband design, enabling invisible integration into cork floor and wall tiles. The compactness, integrability, and stable, high performance of both designs in different operating conditions, make them ideal candidates for IoT applications.

Index Terms-Internet of Things (IoT), Substrate Integrated Waveguide (SIW), Cavity-backed slot antenna, Antenna array, 5GHz Wi-Fi.
\end{abstract}

\section{INTRODUCTION}

The emergence of the Internet of Things (IoT) in the last few years has led to an increasing demand for wireless communication systems that are invisibly integrated inside common objects and that support very high data rates to meet the requirements of various types of future emerging wireless applications [1]. This far-reaching integration and ever increasing demand for higher data rates make antenna design for IoT applications more challenging.

First, an antenna topology that guarantees a stable and high radiation efficiency, when deployed in different environments, and that minimizes detuning, due to varying environmental conditions, is of major importance. Second, a low-cost and low-profile design is essential to facilitate invisible integration into a common object without significantly increasing cost. Furthermore, wideband performance is necessary to meet the stringent requirements of the current and next generation wireless applications. Finally, as multiple input multiple output (MIMO) wireless communication is supported by more and more wireless standards, an antenna topology that allows a compact array design with low mutual coupling is preferable.

Cavity-backed slot (CBS) antennas have the potential to meet these requirements, as they exhibit an excellent suppression of radiation leakage in unwanted directions. In addition, they allow significant size reduction through miniaturization techniques, by relying on the symmetry of the field distribution in the cavity [2]. Moreover, stable and efficient radiation over an ultra-wide bandwidth can be obtained by relying on the excitation of multiple modes [3], whereas the use of substrate integrated waveguide (SIW) technology [4] yields a low-cost and low-profile design. Therefore, in this paper, the CBS antenna topology is combined with SIW technology to realize a new generation of high-performance low-cost antenna designs, facilitating invisible integration inside common objects.

\section{SIW TEChNOLOGY: BenEFITS AND REALIZATION}

SIW technology exhibits very attractive features for IoT applications. Both active and passive components can be implemented on a single substrate by means of a low-cost production process [4], paving the way for a low-profile and cost-effective realization of a complete circuit.

Furthermore, the SIW technology may be combined with innovative substrate materials to obtain a green, stable and high-performance broadband design [2], [3], [4]. For IoT applications, the object's materials can be reused to implement the SIW structure, provided that a dedicated material characterization technique indicates that they possess the appropriate RF characteristics, such as low moisture regain, low losses, and consistent batch-tobatch dielectric permittivity. Such an approach yields a significant cost and area reduction, as the readily available material and area of the object itself are exploited to construct the complete circuit.

Finally, undesired radiation and surface waves are minimized by confining the fields inside the SIW structure through rows of vias that act as effective electric walls. This enables a compact array design with low mutual coupling and significantly improves efficiency, as power is only radiated in the desired directions.

SIW components may be implemented in unconventional materials by means of thin wires in combination with a conductive epoxy sealant or by using tubelets to provide a good electrical contact between the conductive layers on each side of the substrate. The latter method, in combination with copper-plated taffeta as conductive layers (sheet resistance of $0.2 \Omega / \mathrm{sq}$ ), is used in this work. 


\section{SIW CBS ANTENNAS FOR IOT APPLICATIONS}

Two different (multi-)antenna designs, implemented on different substrate materials suitable for IoT applications, and relying on a cavity-backed slot antenna topology, are presented. Both are designed for the $5.15 \mathrm{GHz}$ $5.85 \mathrm{GHz}$ frequency band, referred as the $5 \mathrm{GHz}$ WiFi band in the remainder. The first design is a ultrawideband three-element array, designed for integration into furniture, constructed out of particle board on which a high pressure laminate (HPL) is thermally bonded. The second is a half-mode SIW design optimized for excellent performance when integrated into cork floor and wall tiles, in combination with small dimensions. The remainder of this paragraph describes the operating principles and the design-specific details related to both designs.

\section{A. Three-element SIW CBS Antenna Array for Integration into Furniture}

Particle board, on which HPL is thermally bonded, is a commonly used material to manufacture furniture, such as desks, closets, tables, ... Yet, particle board is not an ideal candidate to be used as the antenna's substrate. Instead, a 3.94-mm-thick closed-cell expanded-rubber by Javaux is adopted as antenna substrate. This material possesses the appropriate charracteristics to obtain a high-performance, broadband design, robust for varying relative humidity levels, and allowing recycling. Its electrical parameters are determined by means of the dedicated material characterization technique described in [5]. It is found that 1.495 and 0.017 are a good estimation for its dielectric permittivity $\left(\epsilon_{r}\right)$ and loss tangent $(\tan \delta)$ at $5.5 \mathrm{GHz}$, respectively. Invisible integration is then obtained by deploying the proposed array inside a recess, milled in the particle board, directly underneath the HPL layer, as shown in Fig. 1 (b).

A prototype of the presented three-element array is depicted in Fig. 1. The array consists of three identical coaxial-fed linearly-polarized SIW CBS antenna elements, each consisting of a rectangular cavity that is split into two subcavities (A and B) by means of a non-resonant rectangular slot. They are arranged in such a way that the array exhibits 3-fold rotational symmetry. This geometry was carefully selected to exploit both spatial and polarization diversity. Each individual antenna element is designed to exhibit a return loss larger than $10 \mathrm{~dB}$ within the complete $5 \mathrm{GHz}$ Wi-Fi band, with $250 \mathrm{MHz}$ margins. Furthermore, mutual coupling remain lower than $-25 \mathrm{~dB}$ over the $[5.15$ 5.85] GHz-band to minimize correlation between received signals. To meet the impedance matching bandwidth criterion, the bandwidth enhancement technique presented in [6], was applied. This means that two different hybrid mode combinations at two neighbouring frequencies are excited and merged, by carefully selecting the dimensions of the slot and cavity. More specifically, the hybrid mode at the lowest frequency is a combination of a weak $\mathrm{TE}_{110}$

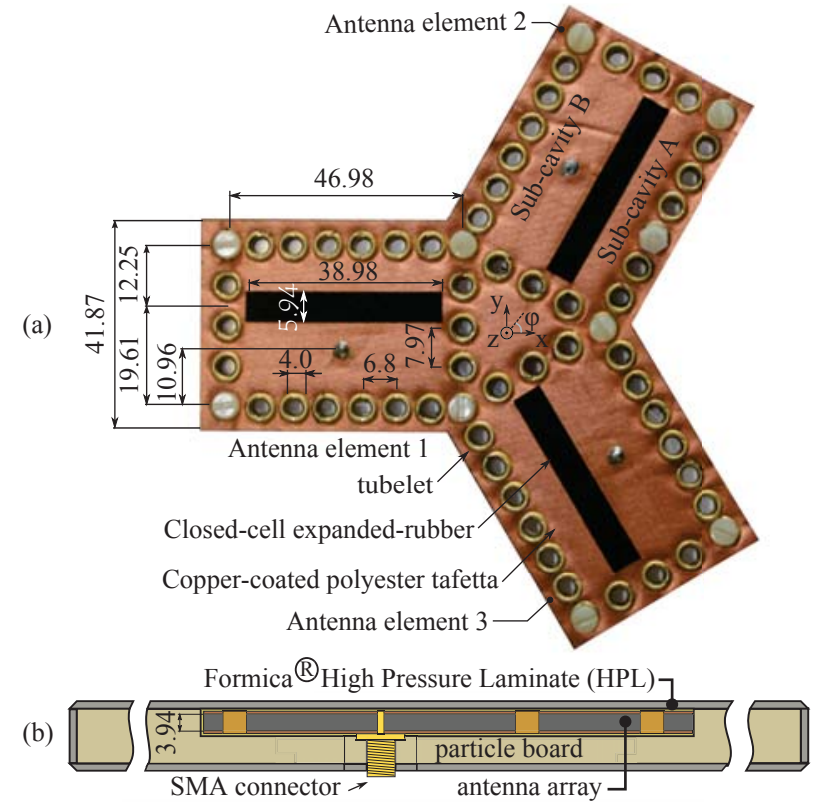

Fig. 1. Three-element SIW cavity-backed slot antenna array for integration into furniture, with annotated dimensions [mm]. (a) Top view of a prototype; (b) Cross-sectional view after integration inside a commonly used board material to manufacture furniture.

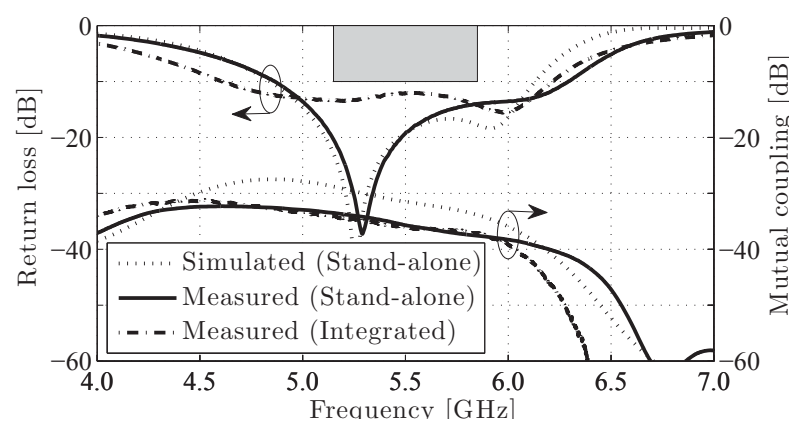

Fig. 2. Return loss and mutual coupling of the three-element SIW CBS antenna array in free-space conditions.

and a strong $\mathrm{TE}_{120}$ mode. This specific combination leads to a dominant field in subcavity B (Fig. 1(a)) that is outof-phase with the fields in subcavity A (Fig. 1(a)). At the highest frequency, the hybrid mode is a combination of a strong $\mathrm{TE}_{110}$ and a weak $\mathrm{TE}_{120}$ mode, yielding a dominant field in subcavity A that is in-phase with the fields in subcavity B. Both specific combinations lead to a large E-field across the slot, causing it to radiate.

The performance of the array was validated in an anechoic chamber by means of Agilent's N5242A PNA$\mathrm{X}$ network analyzer by measuring its $\mathrm{S}$-parameters from 4.0 GHz to $7.0 \mathrm{GHz}$ and its radiation pattern at $5.5 \mathrm{GHz}$, before and after integrating the array according to Fig. 1(b). Fig. 2 shows that the measured stand-alone return loss and mutual coupling are in good agreement with the simulated curves. Fig. 2 also demonstrates that both 


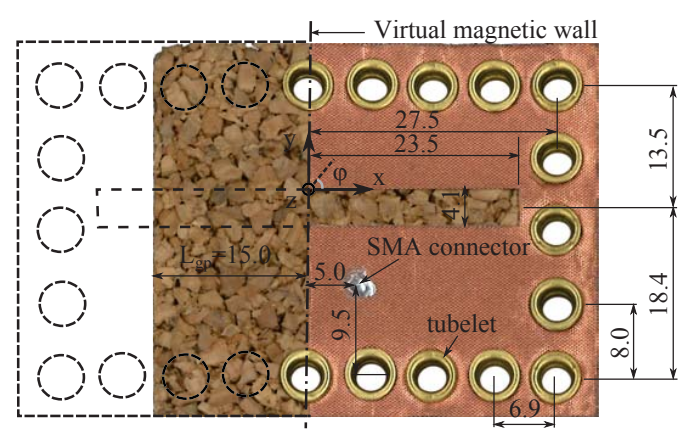

Fig. 3. Prototype of the cork half-mode SIW CBS antenna, with annotated dimensions [mm].

imposed S-parameter requirements remain satisfied after integration of the array. Radiation pattern measurements at $5.5 \mathrm{GHz}$ show that the integration of the array only causes a slight decrease in measured maximum gain (from 5.94 $\mathrm{dBi}$ to $5.80 \mathrm{dBi}$ ) and a neglible decrease in front-to-back ratio (FTBR) (from $15.90 \mathrm{~dB}$ to $15.70 \mathrm{~dB}$ ). In addition, the radiation efficiency equals $78 \%$ when the array is integrated according to Fig. 1(b). Hence, the measurements have proven that the presented array guarantees excellent performance, even after integration.

\section{B. Half-Mode SIW CBS Antenna on Cork Substrate}

The second design [2] is implemented on a 3-mm-thick cork substrate, by Amorim Cork Composites S.A., making it suitable for integration into cork floors or walls. The material exhibits a characterized $\epsilon_{r}$ of 1.22 and $\tan \delta$ of 0.036 at $5.5 \mathrm{GHz}$. Invisible integration in a cork tile is obtained by covering the antenna with a similar cork sheet.

The antenna is based on the same multi-moded CBS antenna topology as described in Subsection III-A. Now, however, the half-mode SIW (HMSIW) technique is applied to reduce the cavity size by half to obtain an even more compact design. Indeed, since both hybrid modes in the initial cavity exhibit a virtual magnetic wall (Fig. 3), the cavity may be cut in half, removing the part for $x<0$ (indicated by dotted lines). Then, the antenna dimensions and feed location are optimized by means of full-wave simulation in CST Microwave studio to obtain a return loss exceeding $10 \mathrm{~dB}$ within the $5 \mathrm{GHz}$ Wi-Fi band with $300 \mathrm{MHz}$ margins. Furthermore, the length of the ground plane $L_{g p}$ is extended to $15.0 \mathrm{~mm}$ to maximize the FTBR.

The HMSIW CBS antenna's performance was then validated in free-space condtions by measuring the antenna's stand-alone reflection coefficient and radiation pattern, and when covered by a 3-mm thick cork sheet (identical to the antenna's substrate). Fig. 4 demonstrates a shift to lower frequencies for the measured reflection coefficients, due to variations in the cork parameters. Covering the antenna by the cork sheet, results in a broader impedance bandwidth because of a downward shift of the lowest hybrid mode. Measuring the radiation pattern at $5.5 \mathrm{GHz}$, shows a comparable maximum gain (from $4.3 \mathrm{dBi}$ to 4.2

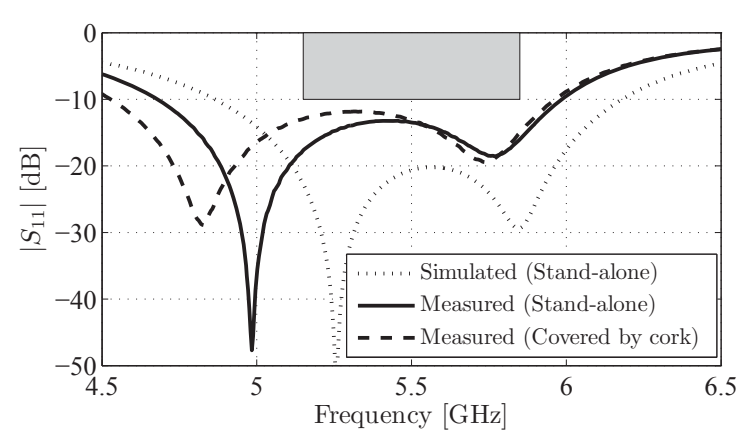

Fig. 4. Return loss of the cork half-mode SIW CBS antenna in free-space conditions.

$\mathrm{dBi}$ ), an increase in FTBR (from $15 \mathrm{~dB}$ to $16.8 \mathrm{~dB}$ ) and a decrease in radiation efficiency (from $85 \%$ to $80 \%$ ), when covering the antenna with the cork sheet. In general, we can conclude that an excellent impedance matching and radiation performance is obtained for both setups.

\section{Conclusion}

In this work, the SIW technology is combined with innovative materials to implement two different high performance, ultra-wideband cavity-backed slot antenna designs to enable invisible integration in an IoT environment, in the mean time guaranteeing stable, high performance. More specifically, two designs were discussed to demonstrate the merits and potential of this design approach: a compact, three-element antenna array with very low mutual coupling for integration into furniture and a miniaturized HMSIW antenna for seamless integration in cork floors and walls. Measurements have been performed and demonstrate excellent performance in free-space and after integration, proving the potential of our approach for IoT applications.

\section{ACKNOWLEDGMENT}

This work was supported in part by the iMinds IoT research program.

\section{REFERENCES}

[1] H. Sundmaeker, P. Guillemin, P. Friess, and S. Woelffl, Eds., Vision and challenges for realising the internet of things. Luxembourg: Publications Office of the European Union, 2010.

[2] O. Caytan, S. Lemey, S. Agneessens, D. Vande Ginste, P. Demeester, C. Loss, R. Salvado, and H. Rogier, "Half-mode substrate-integratedwaveguide cavity-backed slot antenna on cork substrate," Antennas Wirel. Propag. Lett., vol. PP, no. 99, 2015.

[3] S. Lemey and H. Rogier, "SIW textile antennas as a novel technology for UWB RFID tags," in 2014 IEEE RFID-TA, Tampere, Finland, Sept. 8-9, 2014, pp. 256-260.

[4] M. Bozzi and R. Moro, "Low-cost fabrication, eco-friendly materials, and easy integration: the new technological paradigm for the future wireless sensor networks," in European Microwave Conference (EuMC), Nuremberg, Germany, Oct. 6-10, 2013, pp. 858-861.

[5] F. Declercq, H. Rogier, and C. Hertleer, "Permittivity and loss tangent characterization for garment antennas based on a new matrix-pencil two-line method," IEEE Trans. Antennas Propagat., vol. 56, no. 8, pp. 2548-2554, Aug 2008.

[6] G. Q. Luo, Z. F. Hu, W. J. Li, X. H. Zhang, L. L. Sun, and J. F. Zheng, "Bandwidth-enhanced low-profile cavity-backed slot antenna by using hybrid SIW cavity modes," IEEE Trans. Antennas Propagat., vol. 60, no. 4, pp. 1698-1704, Apr 2012. 